\title{
Age-Related Clonal Hematopoiesis
}

National Cancer Institute

\section{Source}

National Cancer Institute. Age-Related Clonal Hematopoiesis. NCI Thesaurus. Code C155821.

The gradual, clonal expansion of hematopoietic stem and progenitor cells carrying specific, disruptive, and recurrent genetic variants, in individuals without clear diagnosis of hematological malignancies. It is associated with an increased risk of developing hematologic cancers. 